\title{
AJS1669, a novel small-molecule muscle glycogen synthase activator, improves glucose metabolism and reduces body fat mass in mice
}

\author{
KAZUHIRO NAKANO $^{1 *}$, SEN TAKESHITA ${ }^{1 *}$, NORIKO KAWASAKI ${ }^{2}$, WATARU MIYANAGA ${ }^{3}$, \\ YORIKO OKAMATSU $^{4}$, MIZUKI DOHI ${ }^{1}$ and TADAKIYO NAKAGAWA ${ }^{5}$
}

\author{
${ }^{1}$ Innovation Promotion Department, Research Institute, EA Pharma Co., Ltd., Kawasaki, Kanagawa 210-868; \\ ${ }^{2}$ Nutrition and Health Science Group Frontier Research Laboratories, Institute for Innovation, Ajinomoto Co., Inc., \\ Kawasaki, Kanagawa 210-8681; ${ }^{3}$ Business Development Department, EA Pharma Co., Ltd., Chuo-ku, Tokyo 104-0042; \\ ${ }^{4}$ Novel Projects Research Group, Institute for Innovation, Ajinomoto Co., Inc., Kawasaki, Kanagawa 210-8681; \\ ${ }^{5}$ Intellectual Property Department, EA Pharma Co., Ltd., Chuo-ku, Tokyo 104-0042, Japan
}

Received August 9, 2016; Accepted February 16, 2017

DOI: $10.3892 /$ ijmm.2017.2909

\begin{abstract}
Impaired glycogen synthesis and turnover are common in insulin resistance and type 2 diabetes. As glycogen synthase (GS) is a key enzyme involved in the synthetic process, it presents a promising therapeutic target for the treatment of type 2 diabetes. In the present study, we identified a novel, potent and orally available GS activator AJS1669 \{sodium 2-[[5-[[4-(4,5-difluoro-2-methylsulfanyl-phenyl) phenoxy] methyl]furan-2-carbonyl]-(2-furylmethyl)amino] acetate\}. In vitro, we performed a glycogen synthase 1 (GYS1) activation assay for screening GS activators and identified that the activity of AJS1669 was further potentiated in the presence of glucose-6-phosphate (G6P). In vivo, we used ob/ob mice to evaluate the novel anti-diabetic effects of AJS1669 by measuring basal blood glucose levels, glucose tolerance and body fat mass index. Repeated administration of AJS1669 over 4 weeks reduced blood glucose and hemoglobin A1c (HbAlc)
\end{abstract}

Correspondence to: Dr Kazuhiro Nakano, Innovation Promotion Department, Research Institute, EA Pharma Co., Ltd., Kawasaki-ku, Kangawaga 2108681, Japan

E-mail: kazuhiro_nakano68@eapharma.co.jp

${ }^{*}$ Contributed equally

Abbreviations: GS, glycogen synthase; GYS1, glycogen synthase 1; G6P, glucose-6-phosphate; GPI, glycogen phosphorylase inhibitor; HbA1c, hemoglobin A1c; HOMA-IR, homeostatic model for assessment of insulin resistance; hGYS1, human GYS1; ${ }^{13} \mathrm{C}-\mathrm{NMR}$, carbon-13 nuclear magnetic resonance spectroscopy; OGTT, oral glucose tolerance test; PPAR, peroxisome proliferator-activated receptor; SGLT2, sodium/glucose co-transporter 2

Key words: glycogen synthase, AJS1669, glucose tolerance, fat mass, mitochondrial fatty acid oxidation levels in $o b / o b$ mice. AJS1669 also improved glucose tolerance in a dose-dependent manner, and decreased body fat mass. The mRNA levels of genes involved in mitochondrial fatty acid oxidation and mitochondrial biogenesis were elevated in skeletal muscle tissue following AJS1669 treatment. Hepatic tissue of treated mice also exhibited elevated expression of genes associated with fatty acid oxidation. In contrast to ob/ob mice, in C57Bl/6 mice AJS1669 administration did not alter body weight or reduce glucose levels. These results demonstrate that pharmacological agents that activate GYS1, the main GS subtype found in skeletal muscle, have potential for use as novel treatments for diabetes that improve glucose metabolism in skeletal muscle.

\section{Introduction}

In 2010, 285 million adults (aged 20 to 79 years) were estimated to have diabetes globally, and this number is expected to reach 439 million by 2030 (1). Currently, although several types of medications used to treat type 2 diabetes have been marketed, pioglitazone and metformin are most commonly used as insulin sensitizers (2).

In humans, skeletal muscle is the major site of glucose uptake and glycogen storage, with both processes being regulated by insulin. Impaired glycogen synthesis is the major abnormality in type 2 diabetes $(3,4)$. For example, carbon-13 nuclear magnetic resonance $\left({ }^{13} \mathrm{C}-\mathrm{NMR}\right)$ spectroscopy in subjects undergoing hyperglycemic-hyperinsulinemic clamping revealed that patients with diabetes have a $50 \%$ or greater decrease in glycogen synthesis relative to healthy individuals (5). In analyses performed at the cellular level, patients with type 2 diabetes involving insulin resistance were found to have decreased glycogen synthase (GS) activity in skeletal muscle $(6,7)$. Insulin sensitivity was found to be enhanced by exercise or weight loss associated with exercise (8-10). Exercise activates GS and enhances insulin sensitivity (11-13). Activation of GS in type 2 diabetes and correction of glucose 
metabolism in skeletal muscle have therefore been regarded as effective approaches to treating diabetes (14).

In skeletal muscle, GS is one of the key enzymes in glucose metabolism. Glucose-6-phosphate (G6P) is converted to UDP-glucose, and GS forms $\alpha$-1,4-glycosidic linkages, whereas branching enzymes create $\alpha-1,6$-glycosidic linkages (15). There are two GS isoforms in mammals, with $69 \%$ homology. One is encoded by the glycogen synthase 2 (GYS2) gene and is expressed only in the liver, whereas the other is encoded by GYS1 and is expressed in the skeletal muscle, brain, kidneys, pancreas and adipose tissue $(16,17)$.

Recently, GS activators have been developed by Roche $(18,19)$. One of the reported compounds was shown to activate GS in the skeletal muscle and liver in a concentration-dependent manner. Repeated administration of this compound in $o b / o b$ mice at a dose of $75 \mathrm{mg} / \mathrm{kg}$ significantly reduced the blood glucose level but did not result in weight gain. We identified AJS1669, as a novel allosteric activator of GYS1. AJS1669 activates human GYS1 (hGYS1) in a concentration-dependent manner. Its activation was found to be additive with G6P, an allosteric activator. AJS1669 accelerated not only glycogen accumulation, but also glycogen degradation in vitro, since the accumulation rate was higher with co-administration of a glycogen phosphorylase inhibitor. In the present study, we selected $o b / o b$ mice for evaluating the effects of this compound on blood glucose level, oral glucose tolerance and $\beta$-oxidation, since AJS1669 has the potential for normalizing blood glucose levels through glycogen metabolism, similar to exercise, and $o b / o b$ mice do not have exercise capacity. We were thus able to exclude the potential effects of exercise and increased skeletal muscle mass in our experimental design. Repeated administration of $10 \mathrm{mg} / \mathrm{kg}$ of AJS1669 over 4 weeks effectively reduced blood glucose level and improved glucose tolerance without excessive body weight gain. Excessive glycogen accumulation in skeletal muscle was not observed, as reflected by in vitro testing. Moreover, fat mass was decreased, and we observed upregulation of genes related to mitochondrial biogenesis in the skeletal muscle. Our results suggest that AJS1669 has potential as a new insulin sensitizer, as it reduces fat mass with upregulation of $\beta$-oxidation through acceleration of glycogen turnover.

\section{Materials and methods}

Chemicals and materials. Sodium 2-[[5-[[4-(4,5-difluoro-2-methylsulfanyl-phenyl)phenoxy]methyl]furan-2-carbonyl]-(2-furylmethyl)amino] acetate (AJS1669) (Fig. 1A) was synthesized by Ajinomoto Pharmaceuticals Co., Ltd. (Kanagawa, Japan). Product purity was over $99 \%$, as determined using a Waters BEH C18 column, $1.7 \mu \mathrm{M}, 2.1 \times 50 \mathrm{~mm}$ (Waters, Milford, MA, USA) and a Waters Acquity UPLC ${ }^{\circledR}$ (ultra-performance liquid chromatography) system, with a mobile phase consisting of acetonitrile: $\mathrm{H}_{2} \mathrm{O}$ including $0.1 \%$ trifluoroacetic acid each. The acetonitrile fraction was increased in a linear fashion from 5 to $95 \%$ over 2 min and kept at $95 \%$ over the next $0.5 \mathrm{~min}$, after which the column was equilibrated to $5 \%$ for $1.5 \mathrm{~min}$.

Pioglitazone was purchased from Tokyo Chemical Industry Co., Ltd. (Tokyo, Japan). Glycogen phosphorylase inhibitor (GPI) was purchased from Millipore (Bedford, MA, USA).
Animals. Male C57BL/6J and ob/ob mice (both 6-weeks of age) were purchased from Charles River Laboratories (Kanagawa, Japan). The mice were fed standard laboratory chow (CRF-1; Charles River, Tokyo, Japan) and tap water ad libitum for the duration of the experiments. All animals were subjected to a reverse 12-h light/dark cycle. All procedures involving the care and use of animals were approved by the Institutional Animal Care and Use Committee of the Pharmaceutical Research Laboratories of Ajinomoto Pharmaceuticals Co., Ltd. prior to the start of experimentation.

In vitro hGYS1 assay. $293 \mathrm{~T}$ cells were obtained from American Type Culture Collection (Manassas, VA, USA). hGYS1 gene cDNA was derived from the muscle cDNA in human MTC Panel I (Takara Bio, Shiga, Japan). The pcDNA 3.1(+) expression vector (Life Technologies Japan, Kanagawa, Japan) containing hGYS1 was transiently transfected into 293T cells. All transfected cells were cultured for 2 days, then washed with phosphate-buffered saline (PBS) and dissolved in lysis buffer consisting of $50 \mathrm{mM}$ Tris- $\mathrm{HCl}(\mathrm{pH} 8.0), 10 \mathrm{mM}$ EDTA, 2 mM EGTA, 100 mM NaF, 1 mM PMSF, 1 mM DTT, and 1X Complete Protease Inhibitor Cocktail Tablet (Roche Diagnostics K.K., Tokyo, Japan). The mixture was homogenized and centrifuged at $16,000 \mathrm{x} \mathrm{g}$ at $4^{\circ} \mathrm{C}$ for $15 \mathrm{~min}$. The precipitated fraction was then reconstituted in lysis buffer and used as a source of the GS enzyme for evaluation after enzyme expression levels were verified by immunoblotting.

The GS (hGYS1) assay was performed as described previously (20). In short, a solution containing AJS1669 at various concentrations with or without $10 \mathrm{mM}$ G6P was added to a polystyrene 96 -well half-area plate (12 $\mu \mathrm{l} /$ well). Next, $18 \mu \mathrm{l}$ of a substrate solution containing $21.6 \mathrm{mM}$ UDP-glucose, $21.6 \mathrm{mM}$ phosphoenolpyruvic acid and $4.05 \mathrm{mM} \mathrm{NADH}$ was added to each well. An enzyme solution containing GS lysates $(0.17 \mathrm{mg} / \mathrm{ml})$ and $1.5 \mu \mathrm{l}$ of a pyruvate kinase/lactate dehydrogenase solution was added (18 $\mu \mathrm{l} /$ well) to prepare a reaction solution. All reagents in the hGYS assay were purchased from Sigma-Aldrich Japan (Tokyo, Japan). After the reaction solution was incubated at $37^{\circ} \mathrm{C}$ for $25 \mathrm{~min}$, the absorbance at $340 \mathrm{~nm}$ was measured using Benchmark Plus (Bio-Rad Laboratories, Tokyo, Japan).

Test compound activity was calculated according to the change in absorbance at $340 \mathrm{~nm}(\Delta \mathrm{A} 340)$. The $\Delta \mathrm{A} 340$ of the reaction solutions containing AJS1669 at a final concentration of $100 \mu \mathrm{M}$ (without any G6P present) or $1 \mu \mathrm{M}$ (with $2.5 \mathrm{mM}$ $\mathrm{G6P})$ was taken as a normalization value (100\%) to calculate the relative activity (\%) at various concentrations. The concentration of the compound that elicited a $50 \%$ increase in relative activity $\left(\mathrm{EC}_{50}\right)$ was calculated using XLfit (IDBS, Tokyo, Japan).

Glucose incorporation into glycogen assay in human muscle cells. Human muscle cells were used after differentiation of SkGM-2 cells (Lonza, Basel, Switzerland). The assay evaluating glucose incorporation into glycogen was performed using previously published methods (21) with slight modification. Briefly, after cells were plated onto a collagen-coated 96-well plate with SkGM-2 containing 10\% fetal bovine serum (FBS) for one day, the cells were subsequently differentiated using serum-reduced SkGM-2 containing 2\% FBS for 3 days. The 
cells were then starved for $4 \mathrm{~h}$ with glucose-free Dulbecco's modified Eagle's medium (DMEM) (Life Technologies Japan) containing $0.1 \%$ bovine serum albumin (BSA). They were then incubated for $3 \mathrm{~h}$ with DMEM containing $0.1 \%$ BSA, $1 \mathrm{~g} / 1$ glucose, and $0.19 \mu / 1\left[{ }^{14} \mathrm{C}\right]$ glucose (Perkin-Elmer Japan, Tokyo, Japan). After washing with ice-cold PBS, cell homogenates were dissolved using $1 \mathrm{~N} \mathrm{NaOH}$ for $10 \mathrm{~min}$ at $60^{\circ} \mathrm{C}$ and transferred to 96-well MultiScreen HTS plates (Merck Millipore, Billerica, MA, USA). Multiscreen plates were washed twice with ice-cold $66 \%(\mathrm{v} / \mathrm{v})$ ethanol and allowed to dry completely. Incorporation of $\left[{ }^{14} \mathrm{C}\right] \mathrm{UDP}$-glucose into glycogen was measured using a scintillation counter. The decay rate (counts/min, cpm) measured in the wells containing AJS1669 at the final concentration of $100 \mu \mathrm{M}$ was taken as $100 \%$ in the calculations of relative activity $(\%)$ after subtracting the cpm of the background wells, which contained dimethyl sulfoxide (DMSO) only.

Assay of GS activity in mouse tissue lysates. Lysates of muscle and liver tissues obtained from mice fasted for $16 \mathrm{~h}$ were homogenized by Microson XL2000 (Qsonica, Newtown, CT, USA) in ice-cold buffer containing $50 \mathrm{mM} \mathrm{KF} \mathrm{(pH} \mathrm{7.0),}$ 10 mM EDTA ( $\mathrm{pH} 7.0$ ), and 10\% glycerol. After centrifugation $(16,000 \mathrm{x} \mathrm{g}$ for $10 \mathrm{~min})$, the supernatant was collected and used as the enzyme solution. The enzyme was incubated 3 times with each studied concentration of test compounds for $30 \mathrm{~min}$ before the GS assay was performed. GS assay was performed using previously described methods (22). Briefly, a $30 \mu \mathrm{l}$ mixture of enzyme and compounds was added to $60 \mu \mathrm{l}$ of an assay mixture containing $0.5 \mu \mathrm{Ci} / \mathrm{ml}\left[{ }^{14} \mathrm{C}\right] \mathrm{UDP}$-glucose (Perkin-Elmer Japan, Kanagawa, Japan). After incubation for $20 \mathrm{~min}$ at $30^{\circ} \mathrm{C}, 75 \mu \mathrm{l}$ of aliquots was immediately spotted onto a filter paper. The filter paper, which completely absorbed the sample, was placed into a vial, washed twice with $10 \mathrm{ml}$ of $66 \%(\mathrm{v} / \mathrm{v})$ ethanol, and completely dried. Incorporation of $\left[{ }^{14} \mathrm{C}\right]$ UDP-glucose into glycogen was measured using a scintillation counter. The decay rate was measured as previously described.

Repeated administration study. Male ob/ob mice (8-weeks of age) were divided into 4 groups $(n=6-8)$. Group allocation was specifically designed so that no significant differences in hemoglobin A1c (HbA1c) level, body weight, and blood glucose level was observed across groups. Mice were orally administered vehicle alone ( $0.5 \%$ methylcellulose), with AJS1669 (3 or $10 \mathrm{mg} / \mathrm{kg})$, or with pioglitazone $(10 \mathrm{mg} / \mathrm{kg})$ twice daily (at 9:00 a.m. and 4:00 p.m.) for 4 weeks. Body weight and food intake were monitored every 2 or 3 days. Blood samples were taken from unrestrained mice by cutting off the tip of the tail. Blood glucose levels were measured every 2 weeks using a Lifecheck sensor (Gunze, Osaka, Japan), and blood HbA1c levels was measured on day 28 (Tosoh, Tokyo, Japan). Body composition was analyzed on day 26 using EchoMRI (EchoMRI LLC, Houston, TX, USA). EchoMRI provides a precise and accurate method for determining the fat and lean tissue content of mice without requiring anesthesia (23). Change in fat mass and lean mass were analyzed by comparing the fat mass before administration and after 4 weeks of treatment. After 4 weeks of treatment, oral glucose tolerance testing (OGTT) was performed following an overnight fast. After OGTT was performed, the mice were fed for another 3 days and then sacrificed. Post-mortem blood and tissue samples were collected for further analysis.

Oral glucose tolerance test. After 4 weeks of compound administration, the mice were fasted overnight. After body weight measurement to determine required administration volumes, glucose (Otsuka, Tokushima, Japan) was orally administered at $1 \mathrm{~g} / \mathrm{kg}$. Blood was collected from the tail vein $1 \mathrm{~min}$ prior to and 30,60 and $120 \mathrm{~min}$ after the administration of glucose, and blood glucose levels were determined using a Lifecheck sensor as described above.

Determination of glycogen content and pancreatic insulin. Frozen tissue samples were weighed and hydrolyzed in $30 \%$ (wt/vol) $\mathrm{KOH}$ solution in a boiling water bath for $30 \mathrm{~min}$, and were agitated vigorously once at $15 \mathrm{~min}$. After cooling on ice, $25 \mu \mathrm{l}$ of $6 \% \mathrm{Na}_{2} \mathrm{SO}_{4}$ and $750 \mu \mathrm{l}$ of $100 \%$ ethanol were added. After overnight storage at $-80^{\circ} \mathrm{C}$, samples were centrifuged at $10,000 \mathrm{x}$ g for $15 \mathrm{~min}$. The glycogen pellet was dried and dissolved in $200 \mu \mathrm{l}$ of water in a water bath at $55^{\circ} \mathrm{C}$, following which $800 \mu \mathrm{l}$ of water was added for a total volume of $1 \mathrm{ml}$. Aliquots $(25 \mu \mathrm{l})$ of resulting glycogen solution were used for subsequent measurements. Glycogen content was determined using a glycogen assay kit (BioVision, Milpitas, CA, USA) according to the manufacturer's instructions. Pancreatic tissue was weighed, homogenized, and extracted with $2 \mathrm{ml}$ of $1.5 \% \mathrm{HCl}-75 \%$ ethanol buffer overnight with shaking in the cold room. For measurements, samples were centrifuged at 3,000 x g for $10 \mathrm{~min}$. The insulin level in the supernatant was measured using an insulin kit (Morinaga Institute of Biological Science, Tokyo, Japan) after a 1:3,000 dilution.

RNA extraction, real-time PCR, and measurement of mitochondrial DNA content. Total RNA was extracted using an RNeasy Plus Mini kit (Qiagen, Tokyo, Japan) according to the manufacturer's instructions. The concentration and purity of total RNA samples were measured using a NanoDrop ND-1000 spectrophotometer (Thermo Scientific, Kanagawa, Japan). cDNA generated via a high capacity cDNA synthesis kit (Life Technologies Japan) was analyzed by quantitative PCR using Power SYBR Premix (Life Technologies Japan). Primer sequences are provided in Table I. Expression data were normalized to the geometric mean of $\beta$-actin levels to control for variability in expression, and were analyzed using the $2^{-\Delta \Delta \mathrm{CT}}$ method. Mitochondrial DNA content was quantified using primers for both NADH dehydrogenase subunit 1 (ND1, an index of mitochondrial DNA) and lipoprotein lipase (LPL, index of genomic DNA). ND1 levels were normalized to LPL DNA content.

Statistical analysis. In this study, data are expressed as the mean \pm SE. Differences among multiple groups (vehicle- vs. AJS1669-treated) and between two groups (vehicle- vs. pioglitazone-treated) were evaluated using step-down Dunnett's multiple comparison test and Student's t-test, respectively, with $\mathrm{p}<0.05$ considered to represent statistically significant differences. All statistical analyses were performed using the EXSUS 8 software package (CAC Exicare Corp., Tokyo, Japan). 
Table I. List of primers used for real-time PCR.

Forward $5^{\prime} \rightarrow 3^{\prime}$

\begin{tabular}{ll}
\hline Gys 1 & TCAGAGCAAAGCACGAATCCAG \\
Ucp 3 & CTGAAGATGGTGGCTCAGGA \\
Tfam & CCTTCGATTTTCCACAGAACA \\
Ppargc $1 \alpha$ & GCGCCGTGTGATTTACGTTG \\
Cpt 1 b & TATCCCAATCATCTGGGTGCTG \\
Acadl & CCAAGAAGAAGTGATTCCTCACCAC \\
Acadm & CAACACTCGAAAGCGGCTCA \\
mt- $N$ d 1 & CCCATTCGCGTTATCTT \\
Lpl & GGATGGACGGTAAGAGTGATTC \\
Gys 2 & ACTGCTTGGGCGTTATCTCTGTG \\
Acoxl & CTGTGGCATTGGCATCGTG \\
Cd36 & GATGGCCTTACTTGGGATTGGA \\
Fabpl 1 & AAGTACCAATTGCAGAGCCAGGA \\
Cpt 1 a & TGTTCAGCTCAGACAGTGGTTCA \\
Ucp 2 & CAGTACCACAGCGCAGGTCA \\
Pckl & GGTGTCATCCGCAAGCTGAA \\
G6pase & GTGCAGCTGAACGTCTGTCTGTC \\
$\beta$-actin & CATCCGTAAAGACCTCTATGCCAAC
\end{tabular}

Reverse $5^{\prime} \rightarrow 3^{\prime}$

CATAGCGGCCAGCGATAAAGA
CCGCAGTACCTGGACTTTCATTA
GCTCACAGCTTCTTTGTATGCTT
CGGTAGGTGATGAAACCATAGCTG
GCGGATGTGGTTCCCAAAG
ACCAATGCCGCCATGTTTCT
ACTTGCGGGCAGTTGCTTG
AAGTTGATCGTAACGGAAGC
ATCCAAGGGTAGCAGACAGGT
ATGCCCGCTCCATGCAGTA
GCAAATCTGATGGCTTTGACTTGA
GGCTTTACCAAAGATGTAGCCAGTG
GGTGAACTCATTGCGGACCA
AGGATCCACCAGGATGCCATA
TCACTACGTTCCAGGATCCCAAG
CTGCTCTTGGGTGATGATGACTG
TCCGGAGGCTGGCATTGTA
ATGGAGCCACCGATCCACA

These primers were used to analyze $\beta$-oxidation and lipid metabolism, mitochondrial DNA content in skeletal muscle, lipid metabolism and glucose metabolism in liver by real-time PCR. Gys 1 , glycogen synthase 1; Ucp3, uncoupling proteins 3; Tfam, transcription factor A, mitochondrial; Ppargcl $\alpha$, peroxisome proliferator-activated receptor $\gamma$, coactivator $1 \alpha$; $C p t 1 b$, carnitine palmitoyltransferase 1B; Acadl, Acyl-CoA dehydrogenase, long chain; Acadm, Acyl-CoA dehydrogenase, medium chain; $m t-N d 1$, NADH dehydrogenase 1 , mitochondrial; Lpl, lipoprotein lipase; Gys2, glycogen synthase 2; Acoxl, acyl-coenzyme A oxidase 1, palmitoyl; Fabpl, fatty acid binding protein 1; Cpt 1a, carnitine palmitoyltransferase 1A; Ucp2, uncoupling protein 2; Pck1, phosphoenolpyruvate carboxykinase 1; G6pase, glucose-6-phosphatase.

\section{Results}

AJS1669, a novel GS activator. Since GYS1 is a key enzyme involved in glycogen metabolism in skeletal muscle, we screened compounds on the basis of their ability to activate hGYS1 in vitro and identified AJS1669 as a novel allosteric activator of hGYS1. AJS1669 activated hGYS1 in a concentration-dependent manner when incubated alone $\left(\mathrm{EC}_{50}=5.2 \mu \mathrm{M}\right)$ (Fig. 1B). A lower concentration of AJS1669 was required to elicit an effect on hGYS1 activity when compared to the concentration-response relationship without G6P $\left(\mathrm{EC}_{50}=0.037 \mu \mathrm{M}\right)$ (Fig. 1C). AJS1669 did not affect hGYS2 in a concentration-dependent manner (data not shown).

Prior to evaluating its action in vivo, we assessed whether AJS1669 has the ability to activate each of the subtypes of peroxisome proliferator-activated receptor (PPAR) using a reporter assay system. Each PPAR ligand-binding domain was overexpressed in CV-1 kidney fibroblast cells derived from African green monkey as a protein fused to the yeast GAL4 DNA-binding domain, and the luciferase activity was measured. AJS1669 did not exhibit any ability to activate PPAR $\alpha, \gamma$ and $\delta$ subtypes (data not shown).

GS activation in mouse tissue lysates and glycogen incorporation in human muscle cells induced by AJS1669. To assess the ability of AJS1669 to activate GS in each organ, mouse skeletal muscle and liver tissue were homogenized and $\left[{ }^{14} \mathrm{C}\right]$ UDP-glucose was used to evaluate GS activity through measurement of ${ }^{14} \mathrm{C}$-labeled glycogen. Intriguingly, AJS1669 exhibited a concentration-dependent activation of GS in the skeletal muscle lysates (Fig. 2A), which was not observed in the liver lysates (Fig. 2B).

Next, to confirm the stimulation of glycogen production by AJS1669 in skeletal muscle tissue, human skeletal muscle cells were incubated with $\left[{ }^{14} \mathrm{C}\right]$ glucose and AJS1669, and the incorporation of $\left[{ }^{14} \mathrm{C}\right]$ glucose into cellular glycogen was measured. Incubation with AJS1669 alone increased the $\left[{ }^{14} \mathrm{C}\right]$ glycogen level in a concentration-dependent manner (Fig. 2C). AJS1669 showed increased stimulation of glycogen production in the presence of glycogen phosphorylase inhibitor (GPI) than in the absence of GPI, whereas GPI alone did not increase the $\left[{ }^{14} \mathrm{C}\right]$ glycogen level (Fig. 2C). These results indicate that the ability of AJS1669 to increase glycogen level is not a consequence of glycogenolysis inhibition, but rather reflects an activation of glycogen synthesis, with AJS1669 upregulating the turnover of glycogen metabolism in the muscle cells by activating both glycogen synthesis and glycogenolysis (Fig. 2C).

Effects of chronic 4-week administration of AJS1669 in normal mice and diabetic ob/ob mice. To confirm the ability of AJS1669 to act as a GYS1 activator with long-term administration, $o b / o b$ mice were administered 3 or $10 \mathrm{mg} / \mathrm{kg}$ of AJS1669 orally twice daily. As a positive control, mice were administered pioglitazone, a PPAR $\gamma$ agonist known to significantly reduce blood glucose levels and improve insulin resistance. The group receiving $10 \mathrm{mg} / \mathrm{kg}$ AJS1669 exhibited a 
<smiles>CSc1cc(F)c(F)cc1-c1ccc(OCc2ccc(C(=O)N(CC(=O)O)Cc3ccco3)o2)cc1</smiles>
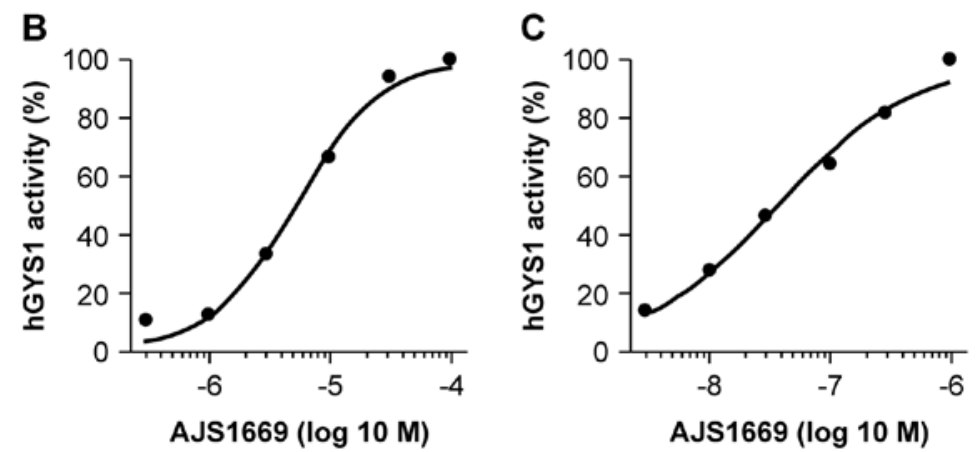

Figure 1. Identification of a novel glycogen synthase activator AJS1669 and its effect on glycogen synthase activity. (A) Chemical structure of AJS1669. (B) Effect of AJS1669 (0.3-100 $\mu \mathrm{M})$ on human glycogen synthase 1 (hGYS1) activity. (C) Effect of AJS1669 (0.03-1 $\mu \mathrm{M})$ on hGYS1 activity in the presence of $2.5 \mathrm{mM}$ glucose-6-phosphate (G6P).
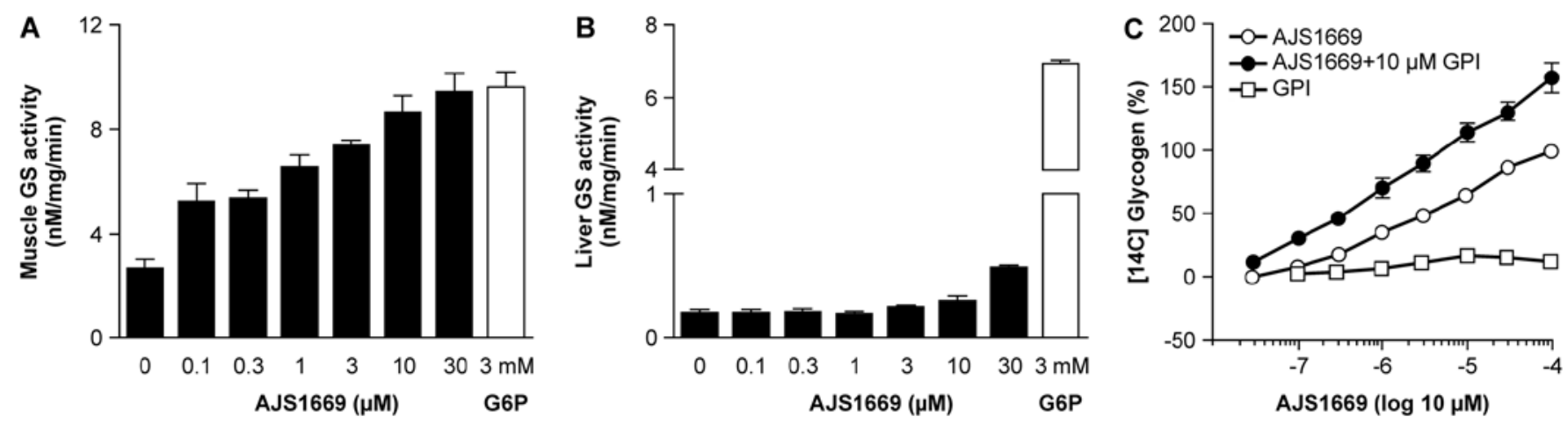

Figure 2. Glycogen synthase activity was determined by incorporation of 2-deoxy-D-glucose into glycogen in mouse tissue lysates and in human muscle cells. (A) Activation of glycogen synthase (GS) in mouse muscle tissue lysates by AJS1669. (B) Activation of GS in mouse liver tissue lysates by AJS1669. (C) Effect of AJS1669 on glycogen accumulation in human muscle cells. Open circles, AJS1669; closed circles, AJS1669 + $10 \mu \mathrm{M}$ glycogen phosphorylase inhibitor (GPI); closed squares, $10 \mu \mathrm{M}$ GPI.

significant decrease in blood glucose level at day 28 (Fig. 3A). Additionally, a reduction in glycated hemoglobin (HbA1c) was observed at day 28 (Fig. 3B). Weight gain over the 4 week administration period was slightly lower in AJS1669-treated animals compared to the vehicle-treated group (Fig. 3C). No major differences in food consumption amount was observed between the treatment groups (Fig. 3D). Next, we measured the plasma level of AJS1669 in samples collected $2 \mathrm{~h}$ after the final administration. Plasma levels were shown to increase linearly in a concentration-dependent manner, with a concentration of $0.92 \pm 0.18 \mu \mathrm{M}$ following administration of the $10 \mathrm{mg} / \mathrm{kg}$ dose (Fig. 3E). The concentration of AJS1669 in skeletal muscle at the same time was determined to be $0.31 \mu \mathrm{M}$ (data not shown).

Effect of repeated administration of AJS1669 on OGTT in ob/ob mice. During the 4th week of AJS1669 administration, mice were administered $1 \mathrm{~g} / \mathrm{kg}$ of D-glucose orally following a 16-h fast. Blood was collected 0,30,60 and 120 min later to measure glucose level. Repeated administration of 3 or $10 \mathrm{mg} / \mathrm{kg}$ AJS1669 induced significant dose-dependent decreases in blood glucose level at $30 \mathrm{~min}$ after administration (Fig. 4A). As a result, AJS1669 was shown to significantly improve glucose tolerance in a dose-dependent manner (Fig. 4B). Fasting blood glucose and insulin level were measured at the same time, and the calculated homeostatic model for the assessment of insulin resistance (HOMA-IR) decreased at higher doses relative to the vehicle-treated group, although there was no significant difference (Fig. 4C).

Chronic effects of AJS1669 on body composition and blood parameters in diabetic ob/ob mice. Glycogen levels in both the skeletal muscle and liver did not significantly increase after 4 weeks of repeated administration of AJS1669 or pioglitazone, 

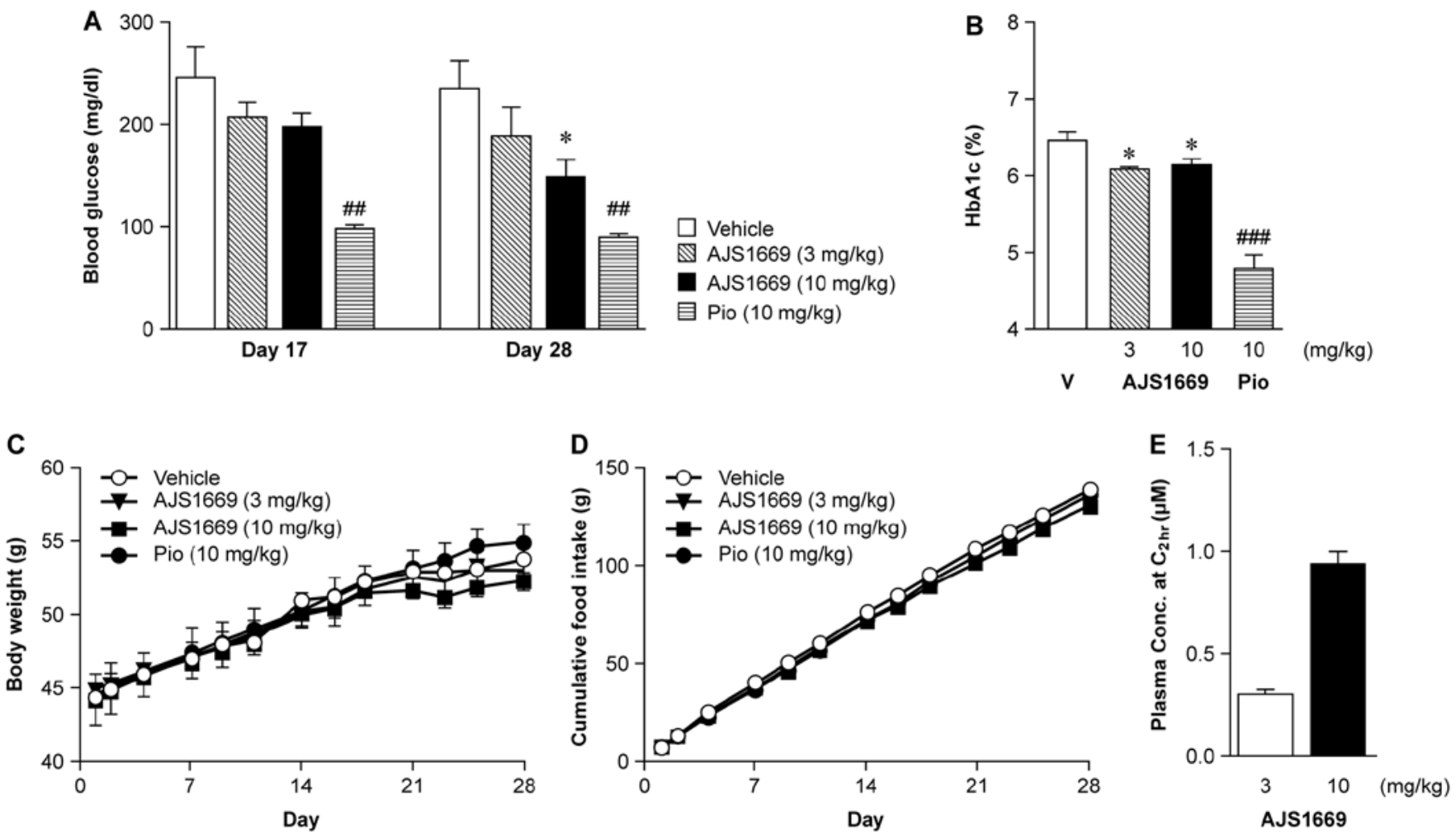

Figure 3. Evaluation of blood glucose and pancreatic parameters after chronic administration of AJS1669 in ob/ob mice. Vehicle, AJS1669, and pioglitazone (Pio) were orally administered twice daily for 4 weeks in wild-type and $o b / o b$ mice (n=6-8). (A) Plasma glucose levels on day 17 and 28 . (B) Blood glycated hemoglobin (HbAlc) levels on day 28. (C) Body weight and (D) cumulative food intake were monitored during treatment. (E) Plasma concentration of AJS1669 after 4 weeks of administration. Data are expressed as the mean \pm SEM. " $p<0.05$ vs. vehicle-treated group, compared using Dunnett's multiple comparison test. ${ }^{\# \#} \mathrm{p}<0.01$ and ${ }^{\# \# \#} \mathrm{p}<0.001$ vs. vehicle-treated group, compared using Student's t-test.
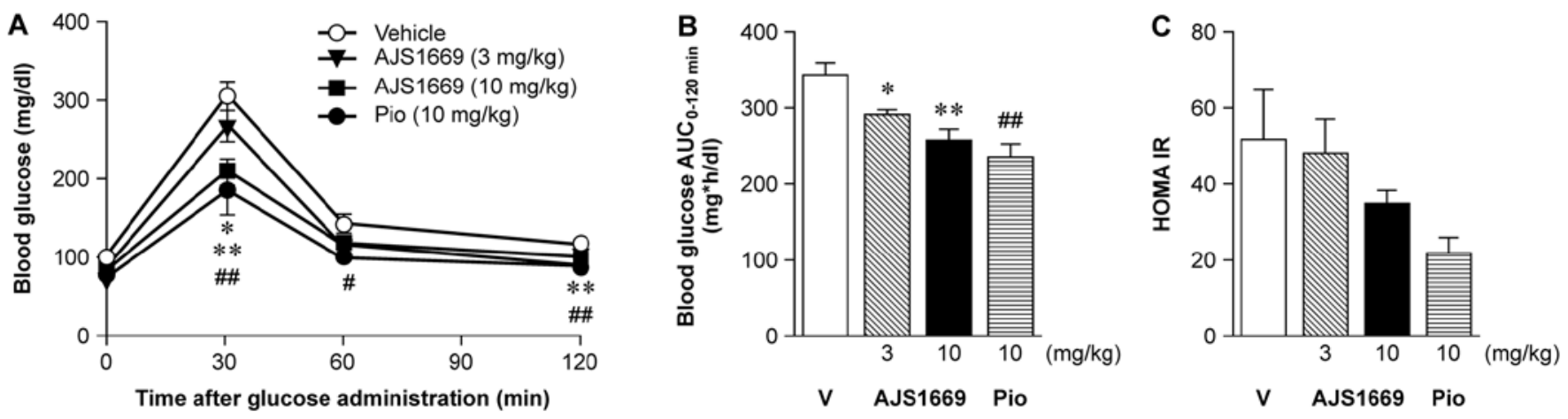

Figure 4. Oral glucose tolerance test after chronic administration of AJS1669, pioglitazone and vehicle in $o b / o b$ mice. (A) Changes in blood glucose level in

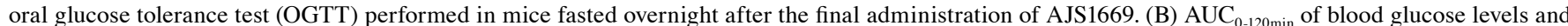
(C) homeostatic model for assessment of insulin resistance (HOMA-IR) calculated using blood samples collected before starting OGTT. Data are expressed as the mean \pm SEM $(n=6-8) . " p<0.05$ and ${ }^{* *} \mathrm{p}<0.01$ vs. vehicle-treated group compared using Dunnett's multiple comparison test; ${ }^{\#} \mathrm{p}<0.05$ and ${ }^{\# \#} \mathrm{p}<0.01$ vs. vehicle-treated group, compared using Student's t-test.

as compared to the levels measured in the vehicle-treated animals (Fig. 5A and B). Insulin content in the pancreas showed a tendency to increase at higher doses (Fig. 5C). Treatment-induced changes in body composition were assessed using EchoMRI, with measurements performed on the day before the start of administration and following 4 weeks of administration. A significant decrease in $\Delta$ fat mass was observed following administration of $10 \mathrm{mg} / \mathrm{kg}$ of AJS1669. Conversely, animals treated with pioglitazone exhibited a significant elevation in $\Delta$ fat mass (Fig. 5D). No difference in $\Delta$ lean mass was observed between the groups (Fig. 5E).

\section{Effect of repeated administration of AJS1669 on mitochondrial} biogenesis in skeletal muscle and hepatic fatty acid oxidation in ob/ob mice. In the skeletal muscle, treatment with AJS1669 elicited no change in Gys 1 mRNA levels. A trend towards a decrease was observed in uncoupling protein $3(U c p 3)$ mRNA levels. However, a significant increase was observed in mRNA 

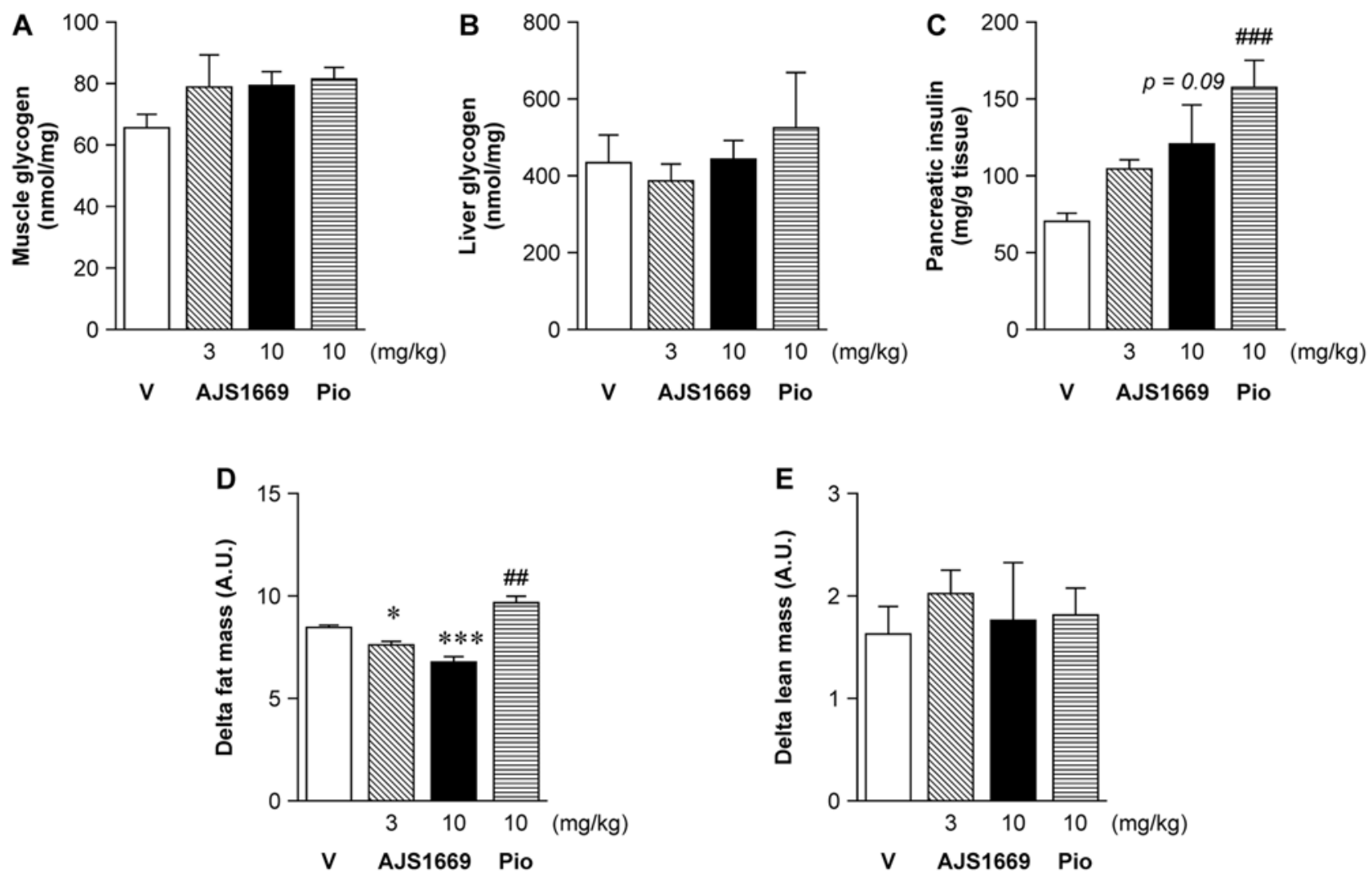

Figure 5. Determination of glycogen amount and body composition by EchoMRI measurement after chronic administration of AJS1669. (A) Muscle glycogen concentration. (B) Liver glycogen level. (C) Pancreatic insulin level. (D) $\Delta$ fat mass calculated using EchoMRI. (E) $\Delta$ lean mass calculated using EchoMRI. Data are expressed as the mean \pm SEM ( $n=6-8)$. ${ }^{*} \mathrm{p}<0.05$ and ${ }^{* *} \mathrm{p}<0.01$ vs. vehicle-treated group, compared using Dunnett's multiple comparison test; ${ }^{\#} \mathrm{p}<0.05$ and ${ }^{\# \#} \mathrm{p}<0.01$ vs. vehicle-treated group, compared using Student's t-test. V, vehicle; Pio, pioglitazone.

Table II. Effect of AJS1669 on liver weight and hepatic functional parameters in $o b / o b$ mice.

\begin{tabular}{lccc}
\hline Treatment & Liver weight $(\mathrm{g})$ & Plasma ALT (IU/l) & Plasma AST (IU/l) \\
\hline Vehicle & $3.89 \pm 0.08$ & $683.29 \pm 134.91$ & $977.57 \pm 206.36$ \\
AJS1669 $(10 \mathrm{mg} / \mathrm{kg})$ & $3.72 \pm 0.71$ & $471.38 \pm 65.39$ & $544.25 \pm 88.32$ \\
Pioglitazone $(10 \mathrm{mg} / \mathrm{kg})$ & $4.33 \pm 0.84$ & $621.00 \pm 116.99$ & $978.00 \pm 268.51$ \\
\hline
\end{tabular}

ALT, alanine aminotransferase; AST, aspartate aminotransferase.

encoding mitochondrial transcription factor A (Tfam), which is involved in the replication and translation of mitochondrial DNA (Fig. 6A). Subsequent evaluation of the mRNA level of genes involved in fatty acid oxidation detected a trend towards elevated levels of carnitine palmitoyltransferase 1B (Cptlb), long chain acyl-CoA dehydrogenase (Acadl), and medium chain acyl-CoA dehydrogenase (Acadm) following AJS1669 treatment. Additionally, AJS1669 administration was observed to increase mitochondrial DNA content in skeletal muscle tissue (Fig. 6B).

The gene expression in the liver was also evaluated. Significant increases in the mRNA levels of genes involved in fatty acid oxidation, including Cd36, Fabpl (which encodes fatty acid binding protein 1), Cptla and Ucp2, were observed (Fig. 6C). No change, however, was observed in the transcription of Pckl and G6pase, which are involved in hepatic gluconeogenesis.

\section{Discussion}

In the present study, we identified AJS1669 as a novel, small-molecule GS activator. In vitro testing showed that this compound activated hGYS1 in a concentration-dependent manner when administered alone, whereas its action was further potentiated in the presence of $2.5 \mathrm{mM}$ of G6P. AJS1669 also exhibited the ability to activate skeletal muscle GS to the same extent as $3 \mathrm{mM}$ G6P in assessment using lysates of mouse skeletal muscle. However, the ability of AJS1669 to activate liver GS was not stronger than its effect on skeletal muscle. AJS1669 acted potently in skeletal muscle and exhibited greater activation with a lower dose in the presence of a high G6P concentration.

To assess the effects at the phenotypic level, skeletal muscle cells were incubated with AJS1669. AJS1669 increased the glycogen level in a concentration-dependent manner. 

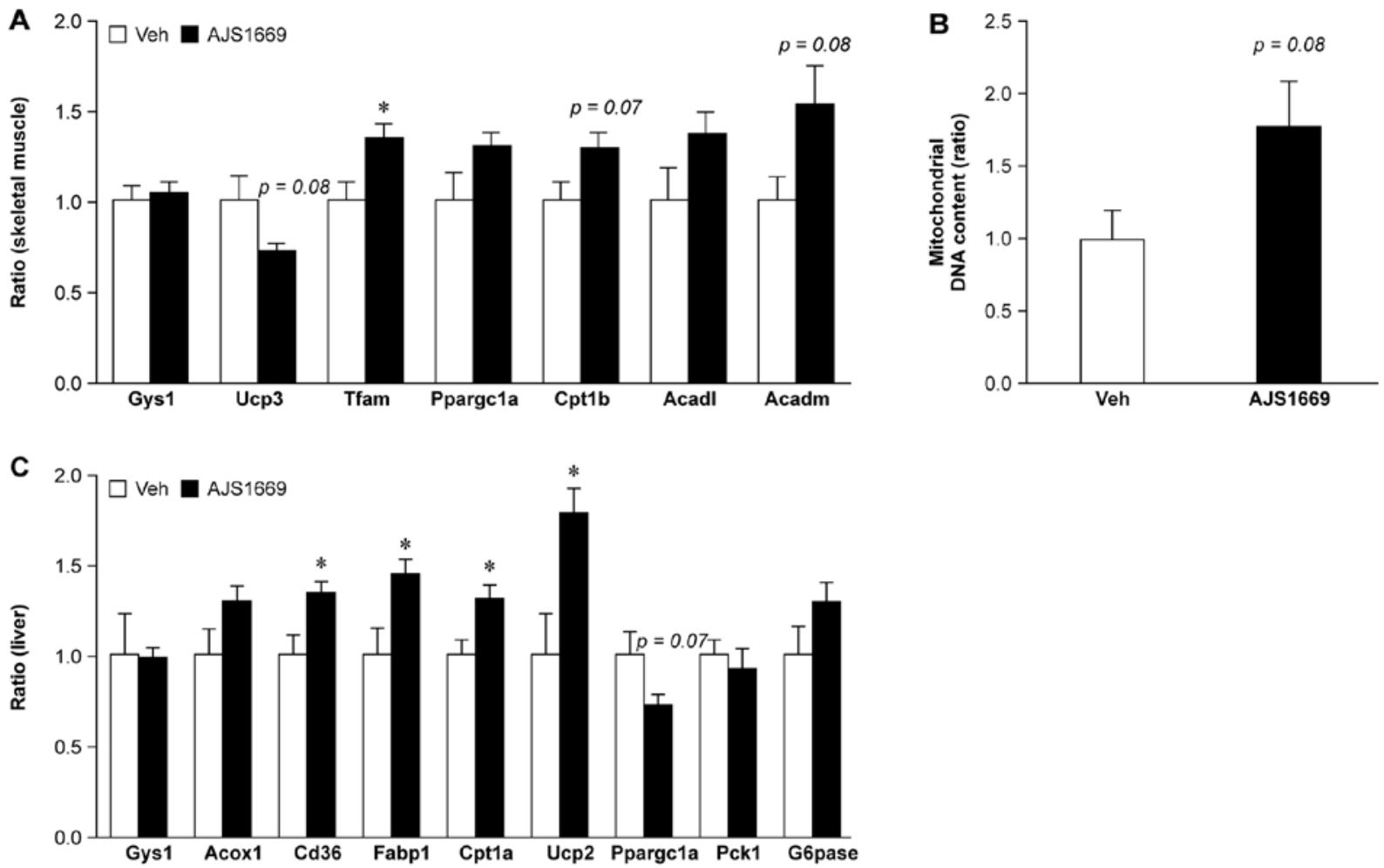

Figure 6. Metabolic gene expression determined by qPCR assay after chronic administration of AJS1669 or vehicle in muscle and liver tissue. (A) Gene expression in mixed gastrocnemius muscle (GC). (B) Mitochondrial DNA content, as assessed by mitochondrial DNA copy number. (C) Gene expression in hepatic tissue. Data are expressed as the mean \pm SEM $(n=7-8)$. ${ }^{*}<<0.05$ vs. vehicle-treated (Veh) group by Student's t-test.

Intriguingly, incubation with AJS1669 and GPI increased glycogen levels to a greater extent than did AJS1669 administered alone. In contrast, no increase was observed following incubation with a GPI alone.

These observations suggest that in addition to promoting glycogen synthesis, AJS1669 caused some glycogen degradation, apparently enhancing the glycogen metabolism turnover. AJS1669 was developed as a hypoglycemic agent for transforming blood glucose into glycogen in both skeletal muscle and liver. However, if AJS1669 can ameliorate insulin resistance as a PPAR agonist, then it could be the first in a new class of insulin sensitizers.

Reportedly, PPAR $\gamma$ and PPARd agonists do not directly activate glycogen synthesis, but rather act to enhance glycogen formation by stimulation of insulin receptors $(24,25)$. We therefore assessed the effect of AJS1669 on the activity of each PPAR subtype. AJS1669 did not elicit any increase in PPAR $\alpha, \gamma$ or $\delta$ activity at a concentration of $100 \mu \mathrm{M}$ (data not shown), which is higher than the physiological level resulting from administration of $10 \mathrm{mg} / \mathrm{kg}$ AJS1669 (Fig. 3E).

To evaluate the pharmacological effect of AJS1669, we measured the concentration of AJS1669 in both the muscle and liver of $o b / o b$ mice. The concentration in the muscle was $0.31 \mu \mathrm{M}$, which was higher than the $\mathrm{EC}_{50}$ of $0.037 \mu \mathrm{M}$ with G6P (Fig. 1C). Based on the potential of AJS1669 for improving glycogen metabolism determined through in vitro tests, we selected $o b / o b$ mice, which are hyperinsulinemic, bulimic, and do not actively exercise. Diabetic-induced mice (DIO) were not used because they are easily affected by food consumption and exercise capacity. Additionally, db/db mice were not used because they may experience body weight loss as a result of hyperglycemia.

Since the AJS1669 concentration of $3 \mathrm{mg} / \mathrm{kg}$ observed in the muscle in vivo was sufficient to activate GYS1 in vitro, and following the 3 Rs policy, we decided to use $10 \mathrm{mg} / \mathrm{kg}$ as the maximum dose for the in vivo experiments. The concentration of AJS1669 was much higher in the liver than that noted in muscle. As previously reported, the furanyl group of AJS1669 has high stability with respect to liver metabolism as it is a 5-membered ring (26). The ability of AJS1669 to reduce blood glucose levels, decrease HbA1c levels, and improve glucose tolerance was observed following 4 weeks of repeated administration (Fig. 4). Interestingly, repeated administration of AJS1669 did not elicit a major change in food consumption. Additionally, 4 weeks of repeated administration of AJS1669 did not elevate the blood lactic acid level (data not shown). No abnormalities were observed in the plasma alanine aminotransferase (ALT) and aspartate aminotransferase (AST) level, which reflect the liver safety profile (Table II). Similarly, C57BL/6 mice treated for 4 weeks with $30 \mathrm{mg} / \mathrm{kg}$ AJS1669 exhibited no changes in body weight, food intake, blood glucose levels, ALT levels, or AST levels (data not shown). AJS1669 therefore does not appear to reduce fat mass by any toxic mechanism.

Intriguingly, although muscle glycogen levels in all animals in the group treated with $10 \mathrm{mg} / \mathrm{kg}$ AJS1669 was increased in comparison with the vehicle-treated group levels, the change was not significant. The same results were observed for pioglitazone-treated animals. Because both AJS1669 and pioglitazone improved glucose tolerance (Fig. 4), 
glycogen levels could be increased by administration of these compounds. In this study, utilizing ad libitum feeding in $o b / o b$ mice, a significant increase in muscle glycogen may not have been detected (Fig. 5). However, AJS1669 increased the glycogen level in muscle cells, and this level was additively increased under treatment with both AJS1669 and GPI (Fig. 2). Therefore, AJS1669 increased not only glycogen accumulation but also glycogen degradation, and this machinery could improve glycogen metabolism. In exercise, both glycogen accumulation and glycogen degradation are involved in energy homeostasis and regulation of insulin sensitivity (27). Since AJS1669 improves the energy capacity of skeletal muscles, it was necessary for us to choose diabetic animals for evaluating this compound. Evaluation of these mice showed that pioglitazone treatment significantly increased the whole body fat mass (a harmful side effect), whereas AJS1669 was observed to markedly decrease the fat mass as we hypothesized (Fig. 5). AJS1669 thus appears to directly induce both glycogen synthesis and glycogen turnover, and therefore may be the first in a new class of insulin sensitizers.

To examine the possibility that the effect of AJS1669 on muscle glycogen causes a metabolic response in muscle, $\beta$-oxidation-related genes were also evaluated (28). Increases in the expression of $\beta$-oxidation-related genes involved in lipid metabolism in the skeletal muscle and liver, as well as in the expression of genes involved in mitochondrial biogenesis, were observed following 4 weeks of repeated administration of AJS1669. Although AJS1669 treatment did not alter the expression of Gysl in skeletal muscle tissue, it significantly increased the expression of Tfam, a gene involved in the stability and transport of mitochondrial gene transcripts. Additionally, a trend towards an increase in the expression of genes involved in $\beta$-oxidation, such as Cptlb and Acadm, was observed following 4 weeks of repeated administration. Regulation of genes involved in mitochondrial biogenesis and fatty acid oxidation by AJS1669 is likely to be associated with a decrease in fat mass. In the liver, no significant modulation of genes controlling gluconeogenesis was observed. As evidenced by the lack of effect on the liver observed through in vitro experiments, AJS1669 may not alter glucose metabolism by directly affecting the liver as excessive liver glycogen accumulation would inhibit the fat mass decrease (29) or reduce body fat mass by attenuating food intake in high-fat-diet model mice (30). In the present study, AJS1669 did not induce accumulation of liver glycogen or decrease fat mass. Following the upregulation of $\beta$-oxidation-related genes involved in lipid metabolism in the skeletal muscle, lipid flow may be altered across the entire body, thus affecting oxidation in the liver.

Although AJS1669 improved glucose homeostasis while decreasing the fat mass in $o b / o b$ mice, this effect was not stronger than the action of pioglitazone. No significant glucose-lowering action was observed at day 17 following administration, and a longer administration period may be required to accurately demonstrate drug efficacy relative to pioglitazone. The physiological mechanism underlying this difference is currently not known, but a number of factors may be involved. AJS1669 could potentially activate GYS1 at a concentration of $<1 \mu \mathrm{M}$ in vitro (Figs. $1 \mathrm{~B}$ and $\mathrm{C}$ and $2 \mathrm{~A}$ ). However, the plasma concentration of AJS1669 at $2 \mathrm{~h}$ after administration was below $1 \mu \mathrm{M}$ (Fig. 3E). The dose of
AJS1669 used in our experiments $(10 \mathrm{mg} / \mathrm{kg})$ may thus not be the optimal dose to elicit the desired pharmacological actions. Moreover, 4 weeks of repeated administration may result in attainment of a homeostatic phase and thus may not be an appropriate term for evaluating the maximum pharmacological effect of this compound. Our aim was to determine the length of time over which administration of AJS1669 can maintain the initial change in glucose metabolism, and we found the duration to surpass 4 weeks. Since fat mass was reduced in AJS1669-treated animals, glucose tolerance was improved, and because the mRNA levels of a number of genes were significantly altered in both skeletal muscle and liver, long-term administration may elicit stronger pharmacological effects. Another factor affecting body composition may be the difference in glycogen metabolism between muscle and liver tissue. In mice, the basal amount of glycogen in the skeletal muscle at satiation is approximately one-tenth of that in the liver, and glycogen metabolism is thought to affect glucose metabolism more significantly in the liver than in the skeletal muscle (31). In humans, however, skeletal muscle accounts for $\sim 40 \%$ of body composition (32) and plays a central role in glucose metabolism (33). In the present study in mouse tissue lysates, AJS1669 elicited a stronger effect in the skeletal muscle than in the liver (Fig. 2A and B), with intense effects not achieved in vivo (Fig. 3). We therefore postulate that the pharmacological effect of AJS1669 observed in mice should be far more pronounced in humans.

In skeletal muscle tissue of patients suffering from type 2 diabetes or obese patients, a decrease in mitochondrial oxidative capacity has been reported $(34,35)$, along with lower expression of genes related to mitochondrial biogenesis $(36,37)$. Increased physical activity and weight loss interventions in these patients were the main inducers of improved mitochondrial functional capacity, and these changes in the mitochondria were associated with improvement of insulin resistance $(38,39)$. Considering the association between GS activity and mitochondrial function, a number of unanswered questions remain regarding the molecules that are directly involved. It is, however, conceivable that repeated administration of AJS1669 may alter glycogen metabolism, and may therefore mimic the effects of exercise and weight loss.

In conclusion, previous studies suggest that beneficial alterations of glycogen metabolism, similar to the physiological effects of physical exercise, may result from attenuation of GS activity in type 2 diabetes. In the present study, AJS1669 enhanced glycogen metabolism and improved mitochondrial biogenesis, and consequently elicited a reduction in body fat mass and exhibited anti-diabetic activity. AJS1669 therefore exhibits potential for use as a new insulin resistance-improving drug with a mechanism of action distinct from that of pioglitazone. AJS1669 can be regarded as a promising candidate compound for the management of type 2 diabetes.

\section{Acknowledgements}

We would like to give special thanks to Atsushi Konishi and Yukio Iino (Ajinomoto Pharmaceuticals Co., Ltd.) for supervising us in this study. Advice given by Yuki Saitoh and Tomomi Yoshida (Ajinomoto Pharmaceuticals Co., Ltd.) has helped immensely with establishing our in vivo and in vitro 
experiments. Kayo Matsumoto and Manami Shuto (Ajinomoto Pharmaceuticals Co., Ltd.) who synthesized and analyzed other types of GS activators, except for AJS1669, provided us with important information. A special note of gratitude is owed to Eri Tabuchi (Ajinomoto Pharmaceuticals Co., Ltd.), who analyzed the 3D structure of human Gys1 protein using information of yeast Gys2 protein and gave us great advice.

\section{References}

1. Shaw JE, Sicree RA and Zimmet PZ: Global estimates of the prevalence of diabetes for 2010 and 2030. Diabetes Res Clin Pract 87: 4-14, 2010.

2. Raskin P: Why insulin sensitizers but not secretagogues should be retained when initiating insulin in type 2 diabetes. Diabetes Metab Res Rev 24: 3-13, 2008

3. Damsbo P, Vaag A, Hother-Nielsen O and Beck-Nielsen H: Reduced glycogen synthase activity in skeletal muscle from obese patients with and without type 2 (non-insulin-dependent) diabetes mellitus. Diabetologia 34: 239-245, 1991.

4. Schalin-Jäntti C, Härkonen M and Groop LC: Impaired activation of glycogen synthase in people at increased risk for developing NIDDM. Diabetes 41: 598-604, 1992.

5. Shulman GI, Rothman DL, Jue T, Stein P, DeFronzo RA and Shulman RG: Quantitation of muscle glycogen synthesis in normal subjects and subjects with non-insulin-dependent diabetes by ${ }^{13} \mathrm{C}$ nuclear magnetic resonance spectroscopy. $\mathrm{N}$ Engl J Med 322: 223-228, 1990.

6. Gaster M, Petersen I, Højlund K, Poulsen P and Beck-Nielsen H: The diabetic phenotype is conserved in myotubes established from diabetic subjects: Evidence for primary defects in glucose transport and glycogen synthase activity. Diabetes 51: 921-927, 2002.

7. Henry RR, Ciaraldi TP, Abrams-Carter L, Mudaliar S, Park KS and Nikoulina SE: Glycogen synthase activity is reduced in cultured skeletal muscle cells of non-insulin-dependent diabetes mellitus subjects. Biochemical and molecular mechanisms. J Clin Invest 98: 1231-1236, 1996.

8. Goodpaster BH,Katsiaras A and Kelley DE: Enhanced fat oxidation through physical activity is associated with improvements in insulin sensitivity in obesity. Diabetes 52: 2191-2197, 2003.

9. Nordby P, Auerbach PL, Rosenkilde M, Kristiansen L, Thomasen JR, Rygaard L, Groth R, Brandt N, Helge JW, Richter EA, et al: Endurance training per se increases metabolic health in young, moderately overweight men. Obesity (Silver Spring) 20: 2202-2212, 2012.

10. Ross R, Dagnone D, Jones PJ, Smith H, Paddags A, Hudson R and Janssen I: Reduction in obesity and related comorbid conditions after diet-induced weight loss or exercise-induced weight loss in men. A randomized, controlled trial. Ann Intern Med 133: 92-103, 2000.

11. Jensen J, Tantiwong P, Stuenæs JT, Molina-Carrion M, DeFronzo RA, Sakamoto K and Musi N: Effect of acute exercise on glycogen synthase in muscle from obese and diabetic subjects. Am J Physiol Endocrinol Metab 303: E82-E89, 2012.

12. Manabe Y, Gollisch KS, Holton L, Kim YB, Brandauer J, Fujii NL, Hirshman MF and Goodyear LJ: Exercise traininginduced adaptations associated with increases in skeletal muscle glycogen content. FEBS J 280: 916-926, 2013.

13. Ryan AS, Ortmeyer HK and Sorkin JD: Exercise with calorie restriction improves insulin sensitivity and glycogen synthase activity in obese postmenopausal women with impaired glucose tolerance. Am J Physiol Endocrinol Metab 302: E145-E152, 2012

14. Abdul-Ghani MA and DeFronzo RA: Pathogenesis of insulin resistance in skeletal muscle. J Biomed Biotechnol 2010: 476279, 2010.

15. Roach PJ, Depaoli-Roach AA, Hurley TD and Tagliabracci VS: Glycogen and its metabolism: Some new developments and old themes. Biochem J 441: 763-787, 2012.

16. Browner MF, Nakano K, Bang AG and Fletterick RJ: Human muscle glycogen synthase cDNA sequence: A negatively charged protein with an asymmetric charge distribution. Proc Natl Acad Sci USA 86: 1443-1447, 1989.

17. Nuttall FQ, Gannon MC, Bai G and Lee EY: Primary structure of human liver glycogen synthase deduced by cDNA cloning. Arch Biochem Biophys 311: 443-449, 1994.
18. Bolin D, Ahmad M, Banner B, Boros LG, Cai J, Gillespie P, Goodnow R, Gubler ML, Hamilton MM, Hayden S et al: A novel approach for the treatment of type 2 diabetes (T2D): characterization of a potent, orally active, small molecule glycogen synthase activator. Presented at 70th ADA Scientific Sessions. Abstract 1389-P, 2010.

19. Qian Y, Bolin D, Conde-Knape K, Gillespie P, Hayden S, Huang KS, Olivier AR, Sato T, Xiang Q, Yun W, et al: Design and synthesis of 2-N-substituted indazolone derivatives as noncarboxylic acid glycogen synthase activators. Bioorg Med Chem Lett 23: 2936-2940, 2013.

20. Danforth WH: Glycogen synthetase activity in skeletal muscle. Interconversion of two forms and control of glycogen synthesis. J Biol Chem 240: 588-593, 1965.

21. Berger J and Hayes NS: A high-capacity assay for activators of glucose incorporation into glycogen in L6 muscle cells. Anal Biochem 261: 159-163, 1998.

22. Thomas JA, Schlender KK and Larner J: A rapid filter paper assay for UDPglucose-glycogen glucosyltransferase, including an improved biosynthesis of UDP- ${ }^{14} \mathrm{C}$-glucose. Anal Biochem 25 : 486-499, 1968.

23. Nixon JP, Zhang M, Wang C, Kuskowski MA, Novak CM, Levine JA, Billington CJ and Kotz CM: Evaluation of a quantitative magnetic resonance imaging system for whole body composition analysis in rodents. Obesity (Silver Spring) 18: 1652-1659, 2010.

24. Dimopoulos N, Watson M, Green C and Hundal HS: The PPARdelta agonist, GW501516, promotes fatty acid oxidation but has no direct effect on glucose utilisation or insulin sensitivity in rat L6 skeletal muscle cells. FEBS Lett 581: 4743-4748, 2007.

25. Sugiyama Y, Taketomi S, Shimura Y, Ikeda H and Fujita T: Effects of pioglitazone on glucose and lipid metabolism in Wistar fatty rats. Arzneimittelforschung 40: 263-267, 1990.

26. St Jean DJ Jr and Fotsch C: Mitigating heterocycle metabolism in drug discovery. J Med Chem 55: 6002-6020, 2012.

27. Jensen J, Rustad PI, Kolnes AJ and Lai YC: The role of skeletal muscle glycogen breakdown for regulation of insulin sensitivity by exercise. Front Physiol 2: 112, 2011.

28. Egan B and Zierath JR: Exercise metabolism and the molecular regulation of skeletal muscle adaptation. Cell Metab 17: 162-184, 2013.

29. Izumida Y, Yahagi N, Takeuchi Y,Nishi M, Shikama A, Takarada A, Masuda Y, Kubota M, Matsuzaka T, Nakagawa Y, et al: Glycogen shortage during fasting triggers liver-brain-adipose neurocircuitry to facilitate fat utilization. Nat Commun 4: 2316, 2013.

30. López-Soldado I, Zafra D, Duran J, Adrover A, Calbó J and Guinovart JJ: Liver glycogen reduces food intake and attenuates obesity in a high-fat diet-fed mouse model. Diabetes 64: 796-807, 2015.

31. Kasuga M, Ogawa W and Ohara T: Tissue glycogen content and glucose intolerance. J Clin Invest 111: 1282-1284, 2003.

32. Clarys JP, Martin AD, Marfell-Jones MJ, Janssens V, Caboor D and Drinkwater DT: Human body composition: A review of adult dissection data. Am J Hum Biol 11: 167-174, 1999.

33. Ivy JL: Role of carbohydrate in physical activity. Clin Sports Med 18: 469-484, v, 1999.

34. Kelley DE, He J, Menshikova EV and Ritov VB: Dysfunction of mitochondria in human skeletal muscle in type 2 diabetes. Diabetes 51: 2944-2950, 2002.

35. Simoneau JA, Veerkamp JH, Turcotte LP and Kelley DE: Markers of capacity to utilize fatty acids in human skeletal muscle: Relation to insulin resistance and obesity and effects of weight loss. FASEB J 13: 2051-2060, 1999.

36. Mootha VK, Lindgren CM, Eriksson KF, Subramanian A, Sihag S, Lehar J, Puigserver P, Carlsson E, Ridderstråle M, Laurila E, et al: PGC-1alpha-responsive genes involved in oxidative phosphorylation are coordinately downregulated in human diabetes. Nat Genet 34: 267-273, 2003.

37. Patti ME, Butte AJ, Crunkhorn S, Cusi K, Berria R, Kashyap S, Miyazaki Y, Kohane I, Costello M, Saccone R, et al: Coordinated reduction of genes of oxidative metabolism in humans with insulin resistance and diabetes: Potential role of PGC1 and NRF1. Proc Natl Acad Sci USA 100: 8466-8471, 2003.

38. Toledo FG, Watkins S and Kelley DE: Changes induced by physical activity and weight loss in the morphology of intermyofibrillar mitochondria in obese men and women. J Clin Endocrinol Metab 91: 3224-3227, 2006.

39. Toledo FG, Menshikova EV, Ritov VB, Azuma K, Radikova Z, DeLany J and Kelley DE: Effects of physical activity and weight loss on skeletal muscle mitochondria and relationship with glucose control in type 2 diabetes. Diabetes 56: 2142-2147, 2007. 